\title{
The effects of progressing and nonprogressing Mycobacterium avium ssp. paratuberculosis infection on milk production in dairy cows
}

\author{
Rebecca L. Smith, ${ }^{* 1}$ Y. T. Gröhn,† A. K. Pradhan,‡ R. H. Whitlock,§ J. S. Van Kessel,\# J. M. Smith,II \\ D. R. Wolfgang, $\Uparrow$ and Y. H. Schukkent** \\ *Department of Pathobiology, University of Illinois College of Veterinary Medicine, Urbana 61802 \\ †Department of Population Medicine and Diagnostic Sciences, Cornell University College of Veterinary Medicine, Ithaca, NY 14850 \\ ‡Department of Nutrition and Food Science and Center for Food Safety and Security Systems, College of Agriculture and Natural Resources, \\ University of Maryland, College Park 20742 \\ §New Bolton Center, Department of Clinical Studies, School of Veterinary Medicine, University of Pennsylvania, Kennett Square 19348 \\ \#Environmental Microbial and Food Safety Laboratory, USDA-Agricultural Research Service, Beltsville, MD 20705 \\ IIDepartment of Animal and Veterinary Sciences, University of Vermont, Burlington 05405 \\ TDepartment of Veterinary and Biomedical Science, Penn State University, University Park 16802 \\ ${ }^{* *}$ GD Animal Health, 7400 AA, Deventer, the Netherlands
}

\begin{abstract}
Longitudinal data from 3 commercial dairy herds in the northeast United States, collected from 2004 to 2011, were analyzed to determine the effect of $M y$ cobacterium avium ssp. paratuberculosis (MAP) infection status and progression path on milk production. Disease status, as indicated by MAP test results, was determined through quarterly ELISA serum testing, biannual fecal culture, and culture of tissues and feces at slaughter. Milk production data were collected from the Dairy Herd Information Association. Animals with positive MAP test results were categorized, based on test results over the full course of the study, as high path (at least one high-positive culture) or low path (at least one positive culture or ELISA). The cumulative numbers of positive ELISA and culture results were recorded. The effects of both MAP infection path, status, and number of positive tests on milk production were analyzed using a mixed linear model with an autocorrelation random effect structure. Low- and high-path animals produced more milk before their first positive test than always-negative animals, especially high-path animals. Although mean production decreased after a first positive test, low-path animals were shown to recover some productivity. High-path animals continued to exhibit a decrease in milk production, especially after their first high-positive fecal culture. These results show that not all animals that test positive for MAP will have long-term production losses. Milk production decreased significantly with each additional positive
\end{abstract}

Received May 15, 2015.

Accepted September 29, 2015.

${ }^{1}$ Corresponding author: rlsdvm@illinois.edu test. Ultimately, production loss appeared to be a function of MAP infection progression.

Key words: paratuberculosis, Johne's disease, milk production

\section{INTRODUCTION}

Johne's disease (JD) is a serious production disease in ruminants that is caused by chronic infection with Mycobacterium avium ssp. paratuberculosis (MAP). It is known to affect milk production (Kudahl et al., 2004; Lombard et al., 2005; Gonda et al., 2007; Nielsen et al., 2009; Smith et al., 2009) and reproduction (Smith et al., 2010). Clinical disease leads to persistent diarrhea, progressive weight loss, and early culling or death (Whitlock and Buergelt, 1996). This has resulted in estimated costs to United States dairy producers of more than $\$ 200$ million per year (Ott et al., 1999).

Economic modeling, based in part on the estimated milk loss associated with infection, has been used to recommend control strategies (Dorshorst et al., 2006; Cho et al., 2012, 2013). Many of these models have assumed that all infections eventually develop into clinical disease. This appears, however, to be inaccurate. According to one longitudinal study, only a small proportion of infected animals ever become clinically affected or high-shedding during their time in the herd (Mitchell et al., 2015a,b). Current opinion on the biology of MAP infection has been shifting in favor of a dual-path approach, in which some infections are self-limiting and others progress to clinical disease. This approach has been used in understanding mycobacterial infections in humans (Meima et al., 1999; Dye and Williams, 2008; Gomes et al., 2012; Liao et al., 2013). Based on this understanding, the effects of MAP infection on milk production might differ between animals progressing to 
clinical disease and animals with self-limiting infections. This could greatly affect the conclusions of economic models, as it might shift test protocols in favor of those able to detect disease progression.

The purpose of this study was to analyze the effect of MAP infection status on milk production in commercial dairy herds using longitudinal data collected from 3 US dairy herds, separating the effect of animals progressing to clinical disease (high path) from those with self-limiting infections (low path). Secondarily, this study considered the usefulness of the number of positive test results as an indicator of the effect of MAP infection status and path combined.

\section{MATERIALS AND METHODS}

The Regional Dairy Quality Management Alliance has collected data from 3 commercial dairy herds (herein, A, B, and C), consisting primarily of Holsteins, in the northeast United States (New York, Pennsylvania, and Vermont) between 2004 and 2010 (Pradhan et al., 2009). Each of these herds is known to be endemically infected with MAP. Herds were visited quarterly to obtain serum samples from all adult animals (those that had calved at least once) and biannually to obtain fecal samples from all adult animals, and all monthly production and health records were obtained through the DHIA. All samples were shipped overnight to the University of Pennsylvania Johne's Laboratory. Additional samples (serum, feces, intestinal epithelium, and intestinal lymph nodes) were collected from a large number of animals sent to slaughter and most animals dying on-farm during the study period (300 of the 1,053 animals culled or dead). These samples were refrigerated on-site and shipped to the laboratory within $1 \mathrm{~d}$ of collection. Serum samples were tested by using the ParaChek ELISA (Prionics USA Inc., La Vista, NE) for antibody reactions to MAP antigens, and results were reported as positive or negative. Fecal and tissue samples were tested by 4-tube culture for presence of viable MAP organisms, and results were reported as colony-forming units per tube.

For the model, all adult cows were assigned a constant MAP infection path (MAPpath) based on all test results over the course of the study. One of 3 MAPpath categories was assigned: (1) negative - all tests were negative throughout study; (2) low-at least 1 positive test during study, either fecal culture (FC) with $<50 \mathrm{cfu} /$ tube, ELISA, or postmortem tissue culture; or (3) high - at least $1 \mathrm{FC}$ or tissue culture with $>50$ cfu in at least 1 tube during study). Infection status (MAPstatus) was a time-dependent variable defined for each observation as previously described (Smith et al., 2009), and was divided into 4 categories: negative, latent, low shedding, and high shedding. Observations for negative-path cows were defined as negative for MAPstatus. Observations for low- and high-path cows that occurred before a positive diagnostic test were defined as latent. Observations for low- and high-path cows that occurred after a positive diagnostic test and, in the case of high-path cows, before a high-positive diagnostic test, were defined as low shedding. Observations for high-path cows that occurred after a diagnostic test with $>50 \mathrm{cfu}$ in at least 1 tube were defined as high-shedding. Therefore, for example, an animal with a low-positive FC during its second lactation and a highpositive FC during its third lactation would be defined as MAPpath "high" throughout the study, but would have MAPstatus defined as "latent" throughout its first lactation, becoming "low" after the positive FC during its second lactation, and then becoming "high" after the high-positive FC during its third lactation. An animal with a low-positive FC during its second lactation and a high-positive tissue culture at slaughter would be defined as MAPpath "high" throughout the study and would have MAPstatus defined as "latent" during its first lactation and "low" for the remainder of its life. Observations for cows with only 1 or 0 test results were removed from the analysis if the results were negative due to assumed lack of diagnostic sensitivity; animals with multiple negative test results were included in the analysis. For all low and high path cows, the variable $M$ thP was created to indicate the number of months spent in the study at a particular status level; $M t h P$ was set equal to zero for MAPpath-negative animals.

Parity was divided into first and later lactations to account for the different shape of the lactation curve in first-parity animals, and Wilmink's correction (Wilmink, 1987) was used to model the lactation curve with an interaction with parity. A variable for test-day number (TDnr) was created to indicate the order of milk test-days for a given animal in the study. Daily milk production $(\mathrm{kg})$, somatic cell linear score (LS, the log of the SCC), and DIM in the current lactation were used as reported by DHIA, removing all observations with non-numeric or zero values for milk and LS. Season was categorized as winter (January to March), spring (April to June), summer (July to September), and fall (October to December).

Mixed model analysis was performed using the gls function of the nlme package in $\mathrm{R}$, implemented through Revolution R Enterprise (Revolution Analytics Inc., Mountain View, CA). Parity, LS, calving season, and herd were included as fixed effects. Cow was included as a random variable, with first-order autocorrelation by $T D n r$. The model structure was 


$$
\begin{aligned}
\text { Milk }_{i t} & =\beta_{0}+\beta_{1, p} \text { parity }_{i t}+\beta_{2} L_{i t}+\beta_{3, s} \text { season }_{i t} \\
& +\beta_{4} \text { Herd }_{i}+\beta_{5} \text { DIM }_{i t}+\beta_{6} \exp \left[-0.1 \times D I M_{i t}\right] \\
& +\delta_{1, p} \text { DIM }_{i t} \times \text { parity }_{i t}+\delta_{2, p} \exp \left[-0.1 \times \text { DIM }_{i t}\right] \\
& \times \text { parity }_{i t}+\beta_{7, n} \text { MAPpath }_{i}+\beta_{8, m} \text { MAPstatus }_{i t} \\
& +\beta_{9} \text { MthP }_{i t}+\rho_{i} \varepsilon_{i, t-1}+u_{i t p s m n},
\end{aligned}
$$

where the outcome $\left(M_{i l k}\right)$ is milk production $(\mathrm{kg}), i$ indicates cow, $t$ indicates milk test-day, the $\beta_{k}$ are the effects of the $k$ outcomes, and the $\delta_{j}$ are the effects of the $j$ interactions. The term $\rho_{i} \varepsilon_{i, t-1}$ provides the first-order autocorrelation between milk test-days $t$ in individual cows $i$, and $u_{i t p s m n}$ is the error term for each observation, where $i$ is the individual cow, $t$ is the milk test-day, $p$ is the parity, $s$ is the season, $m$ is the JD status, and $n$ is the JD path. All other parameters are as defined above. Effects were considered significant at the $\alpha=0.05$ level. All possible interactions between MAPpath, MAPstatus, and MthP were considered in stepwise fashion.

To compare the results directly to previous analysis, the data were also analyzed using a model lacking the MAPpath variable. The model structure was

$$
\begin{aligned}
\text { Milk }_{i t} & =\beta_{0}+\beta_{1, p} \text { parity }_{i t}+\beta_{2} L S_{i t}+\beta_{3, s} \text { season }_{i t} \\
& +\beta_{4} \text { Herd }_{i}+\beta_{5} \text { DIM }_{i t}+\beta_{6} \exp \left[-0.1 \times D I M_{i t}\right] \\
& +\delta_{1, p} \text { DIM }_{i t} \times \text { parity }_{i t}+\delta_{2, p} \exp \left[-0.1 \times D I M_{i t}\right] \\
& \times \text { parity }_{i t}+\beta_{7, m} \text { MAPstatus }_{i t}+\beta_{8} \text { MthP }_{i t} \\
& +\left(\rho_{i} \varepsilon_{i, t-1}+u_{i t p s m}\right),
\end{aligned}
$$

where all variables and procedures were as defined above.

To compare results to other potential test results, the data were also analyzed based on the cumulative number of positive ELISA results (Epos) and the cumulative number of positive FC results (FCpos). The structure of the model for the ELISA results, where $M t h P$ was not included as the shedding status of the animal would not be known, was

$$
\begin{aligned}
\text { Milk }_{i t} & =\beta_{0}+\beta_{1, p} \text { parity }_{i t}+\beta_{2} L S_{i t}+\beta_{3, s} \text { season }_{i t} \\
& +\beta_{4} \text { Herd }_{i}+\beta_{5} \text { DIM }_{i t}+\beta_{6} \exp \left[-0.1 \times D I M_{i t}\right] \\
& +\delta_{1, p} \text { DIM }_{i t} \times \text { parity }_{i t}+\delta_{2, p} \exp \left[-0.1 \times D I M_{i t}\right] \\
& \times \text { parity }_{i t}+\beta_{7, m} \text { Epos }_{i t}+\left(\rho_{i} \varepsilon_{i, t-1}+u_{i t p s m}\right) .
\end{aligned}
$$

The structure of the model for the FC results was

$$
\begin{aligned}
\text { Milk }_{i t}= & \beta_{0}+\beta_{1, p} \text { parity }_{i t}+\beta_{2} L S_{i t}+\beta_{3, s} \text { season }_{i t} \\
& +\beta_{4} \text { Herd }_{i}+\beta_{5} \text { DIM }_{i t}+\beta_{6} \exp \left[-0.1 \times D I M_{i t}\right] \\
& +\delta_{1, p} \text { DIM }_{i t} \times \text { parity }_{i t}+\delta_{2, p} \exp \left[-0.1 \times D I M_{i t}\right] \\
& \times \text { parity }_{i t}+\beta_{7} \text { FCpos }_{i}+\beta_{8} \text { MthP }_{i t}+\beta_{9} \text { Fpos }_{i} \\
& \times \text { MthP }_{i t}+\rho_{i} \varepsilon_{i, t-1}+u_{i t p s m n} .
\end{aligned}
$$

Models were compared using Bayesian information criterion (BIC) values. All analyses were performed using the nlme package (Pinheiro et al., 2015) in $\mathrm{R}$ version 3.0.3 (The R Development Core Team, 2014).

\section{RESULTS}

A description of the data available for analysis is shown in Table 1. Herd A had an initial FC prevalence of 0.03 and a final FC prevalence of 0 (range 0 to 0.03 ), herd B had an initial FC prevalence of 0.07 and a final FC prevalence of 0 (range 0 to 0.07 ), and herd $\mathrm{C}$ had an initial FC prevalence of 0.14 and a final FC prevalence of 0.02 (range 0.01 to 0.14 ). In total, 31,735 observations were available for analysis, representing 1,669 individual cows. Overall, 472 observations (1.5\% of all valid observations) from 173 animals were deleted because they lacked a sufficient number of test results, having either no test results or only 1 negative test result. Herds $\mathrm{A}$ and $\mathrm{C}$ had observations in all path and status combinations, but herd B contained only 1 high-path animal and that animal was diagnosed at slaughter. There were no indications that mean DIM or parity differed by path or status.

Low-path animals spent (median) 14.2 mo (range: 0 to 63.7 ) in latent status and 13.5 mo (range: 0 to 56.7) in low-shedding status. High-path animals spent 12.9 mo (range: 0 to 57.3) in latent status, 9.2 mo (range: 2.3 to 22.4 ) in low-shedding status, and 4.5 mo (range: 0.1 to 15.1 ) in high-shedding status. All distributions of time spent in status-path combinations were rightskewed.

The results of the statistical analyses based on all test combinations are shown in Table 2. All predictors were highly significant. The model with the MAPpath variable had a BIC value of 213,918.1, compared with a BIC value of $215,847.8$ for the model without the MAPpath variable, indicating strong preference for the model including path. The MAPpath variable, the MAPstatus variable, the MAP time variable, and the status $\times$ time interaction were all significant. The difference in daily milk production between test-negative 


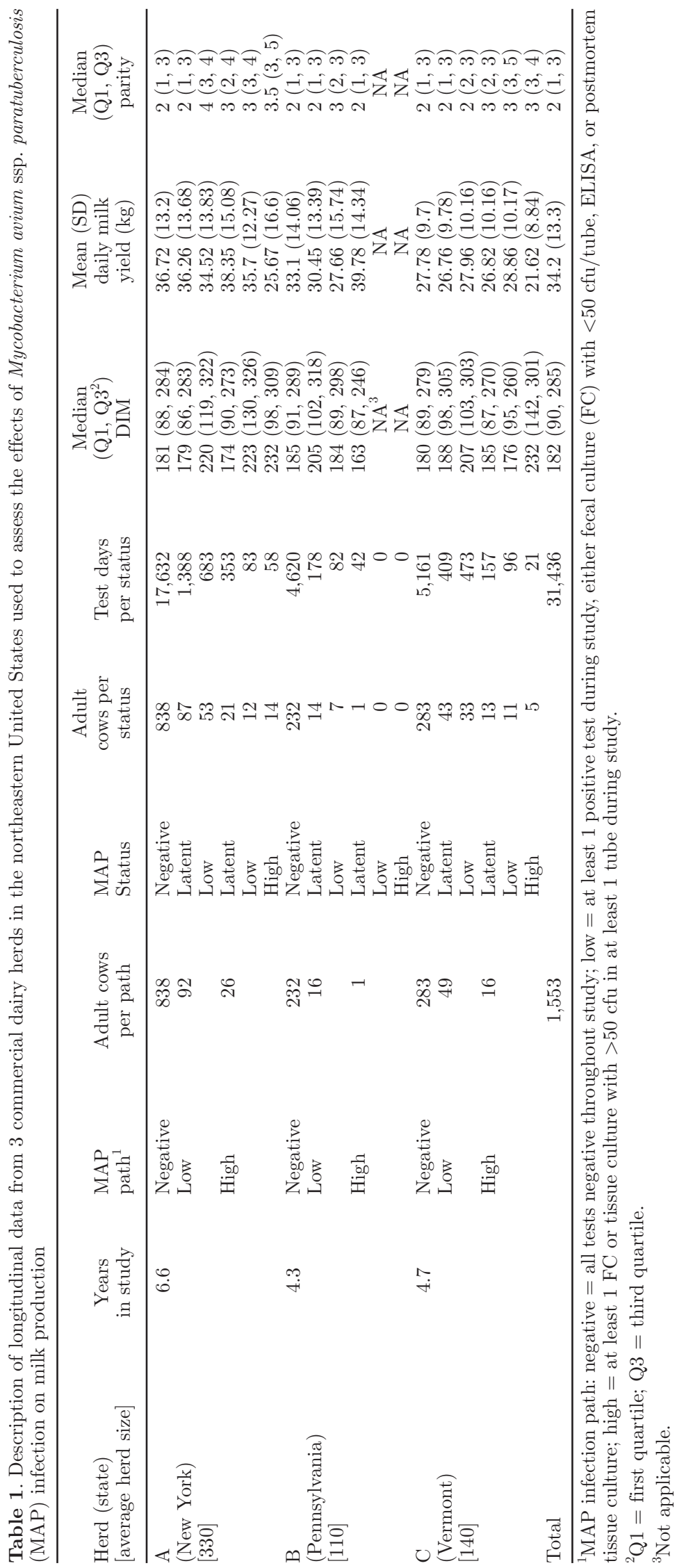


Table 2. Results of a linear mixed model for daily milk production (kg) based on combined results of ELISA and fecal culture tests for Mycobacterium avium ssp. paratuberculosis (MAP)

\begin{tabular}{|c|c|c|c|c|c|}
\hline \multirow[b]{2}{*}{ Variable } & \multirow[b]{2}{*}{ Class } & \multicolumn{2}{|c|}{ With path ${ }^{1}$} & \multicolumn{2}{|c|}{ Without path ${ }^{1}$} \\
\hline & & Parameter & $P$-value & Parameter & $P$-value \\
\hline $\begin{array}{l}\text { Parity } \\
\text { (base = first lactation) }\end{array}$ & $>1$ lactation & 9.60 & $<0.001$ & 9.73 & $<0.001$ \\
\hline $\operatorname{DIM}_{-0.1 \times \text { DIM }}$ & & -0.02 & $<0.001$ & -0.02 & $<0.001$ \\
\hline $\mathrm{e}^{-0.1 \times \mathrm{DIM}}$ & & -27.28 & $<0.001$ & -27.82 & $<0.001$ \\
\hline $\begin{array}{l}\mathrm{e}^{-0.1 \times \text { DIM }} \times \text { Parity } \\
(\text { base }=\text { first lactation })\end{array}$ & $>1$ lactation & 0.29 & 0.79 & 0.80 & 0.44 \\
\hline Linear score & & -0.98 & $<0.001$ & -0.98 & $<0.001$ \\
\hline Herd & B & -3.93 & $<0.001$ & -3.93 & $<0.001$ \\
\hline$($ base $=\mathrm{A})$ & $\mathrm{C}$ & -10.69 & $<0.001$ & -10.65 & $<0.001$ \\
\hline Season & Spring & 2.60 & $<0.001$ & 2.59 & $<0.001$ \\
\hline$($ base $=$ fall $)$ & Summer & 1.46 & $<0.001$ & 1.45 & $<0.001$ \\
\hline negative for model without path) & High-shedding & NA & NA & -3.96 & $<0.01$ \\
\hline MAP time $(M t h P)$ & & -0.80 & $<0.01$ & -0.79 & $<0.01$ \\
\hline MAP time $\times$ MAP status & Latent & 0.72 & $<0.01$ & 0.71 & $<0.01$ \\
\hline (base = high-shedding) & Low-shedding & 0.89 & $<0.001$ & 0.87 & $<0.001$ \\
\hline
\end{tabular}

${ }^{1}$ MAP infection path: negative $=$ all tests negative throughout study; low $=$ at least 1 positive test during study, either fecal culture $(\mathrm{FC})$ with $<50 \mathrm{cfu} /$ tube, ELISA, or postmortem tissue culture; high = at least $1 \mathrm{FC}$ or tissue culture with $>50$ cfu in at least 1 tube during study.

${ }^{2}$ Not applicable.

and test-positive animals is shown in Table 3 and, using simulated data for representative cows in herd A and results from the model with the MAPpath variable, in Figure 1. In both models, milk production was, on average, higher in latent animals and lower in low-positive animals than in test-negative animals, but the effect was strongest in high-path animals. Milk production decreased as animals approached their first positive test. Without the MAPpath variable, milk production decreased on average for every month since testing positive. With the MAPpath variable added, recovery in milk production was noted after the first positive test in low-path animals. In both models, milk produc- tion was greatly decreased after a high-positive test and continued to decrease over time.

The results of the statistical analyses based on the cumulative number of positive tests are shown in Table 4. The model based on FC results had a BIC value of $182,205.7$, compared with a BIC value of $188,180.7$ for the model based on ELISA results, indicating that the model based on FC results was strongly preferred to any other model. The MAP path variable was highly significantly correlated with the total number of positive tests an animal had during its time in the study (either ELISA, $\rho=0.57$, or FC, $\rho=0.60$, where $\rho$ is the Spearman correlation coefficient). In both models,

Table 3. Difference in estimated daily milk production from cows always test-negative for Mycobacterium avium ssp. paratuberculosis (MAP), based on path and status ${ }^{1}$

\begin{tabular}{|c|c|c|c|}
\hline MAP path ${ }^{2}$ & MAP Status & Difference with path $(\mathrm{kg})$ & Difference without path $(\mathrm{kg})$ \\
\hline \multirow[t]{2}{*}{ Low } & Latent & $1.3-0.08 \times M \operatorname{th} P$ & $1.5-0.08 \times M \operatorname{th} P$ \\
\hline & Low & $-1.6+0.09 \times M \operatorname{th} P$ & $-1.4-0.09 \times M t h P$ \\
\hline \multirow[t]{3}{*}{ High } & Latent & $2.3-0.08 \times M t h P$ & $1.5-0.08 \times M \operatorname{th} P$ \\
\hline & Low & $-0.5+0.09 \times M \operatorname{MthP}$ & $-1.4-0.09 \times M \operatorname{th} P$ \\
\hline & High & $-3.9-0.08 \times M t h P$ & $-4.0-0.7 \times M \operatorname{th} P$ \\
\hline
\end{tabular}

${ }^{1}$ The models include a time-dependent covariate, $M$ thP, to represent months at current status.

${ }^{2} \mathrm{MAP}$ infection path: negative $=$ all tests negative throughout study; low $=$ at least 1 positive test during study, either fecal culture (FC) with $<50 \mathrm{cfu} /$ tube, ELISA, or postmortem tissue culture; high = at least $1 \mathrm{FC}$ or tissue culture with $>50 \mathrm{cfu}$ in at least 1 tube during study. 


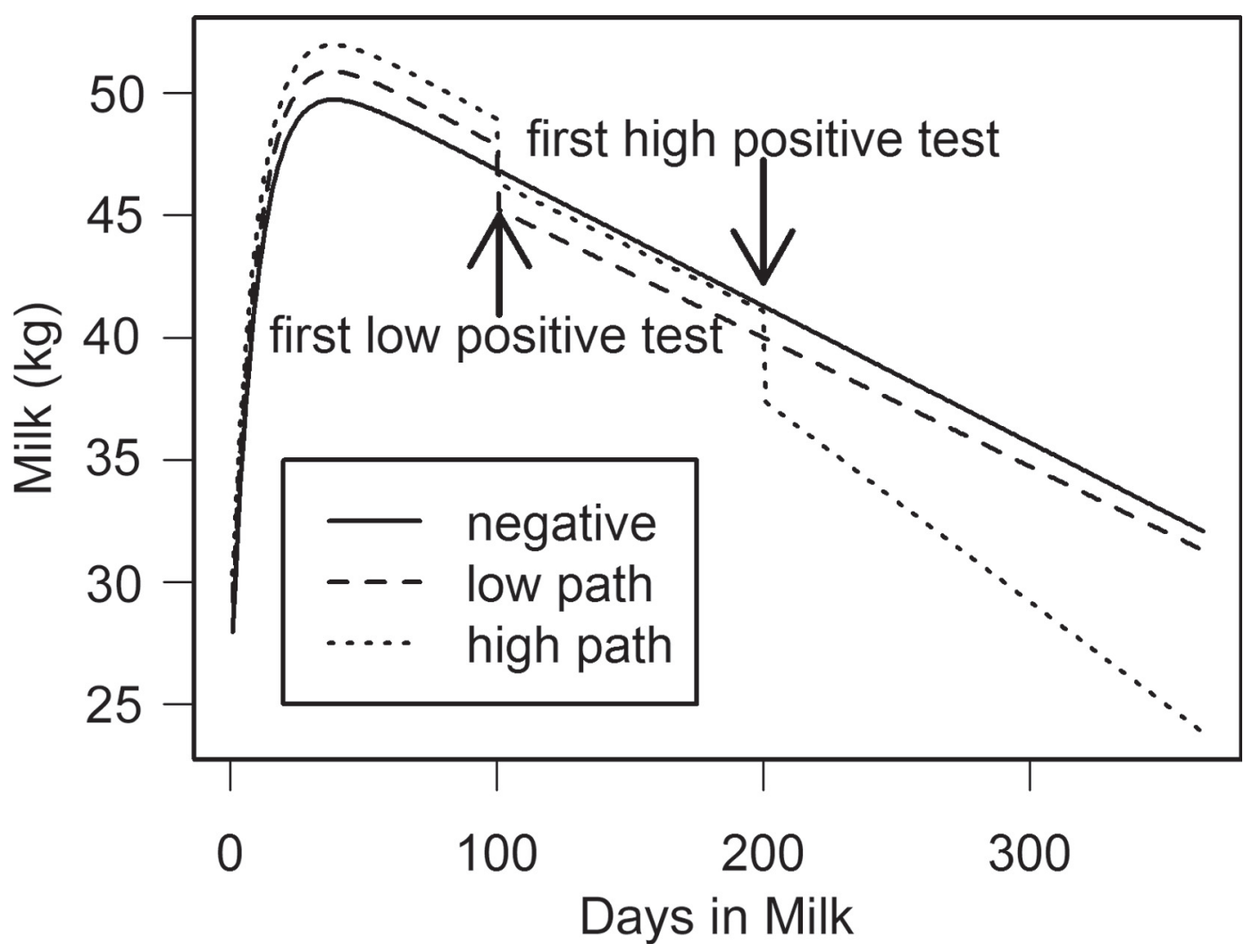

Figure 1. Predicted milk yield $(\mathrm{kg})$ for an average cow in herd A in the third lactation, comparing a test-negative animal (solid line) with test-positive animals in the low path (dashed line) and high path (dotted line). Mycobacterium avium ssp. paratuberculosis infection path: negative $=$ all tests negative throughout study; low $=$ at least 1 positive test during study, either fecal culture $(\mathrm{FC})$ with $<50 \mathrm{cfu} /$ tube, ELISA, or postmortem tissue culture; high $=$ at least $1 \mathrm{FC}$ or tissue culture with $>50 \mathrm{cfu}$ in at least 1 tube during study). The test-positive animals were simulated to become low-positive at 100 DIM, and the high-path animal was simulated to become high-positive at 200 DIM. The model including path as a variable [Eq. 1] was used to predict values.

Table 4. Results of a linear mixed model for daily milk production $(\mathrm{kg})$ based on number of positive results of ELISA or fecal culture tests for Mycobacterium avium ssp. paratuberculosis (MAP)

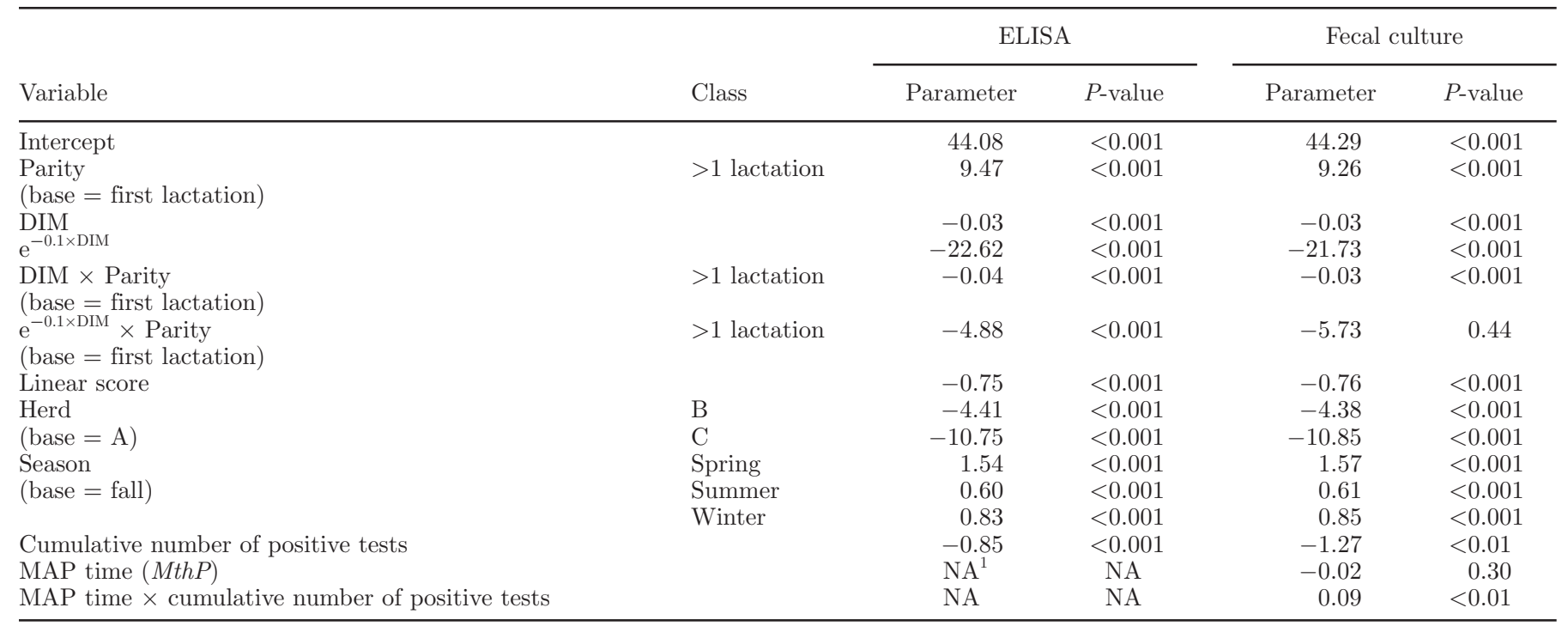

${ }^{1}$ Not applicable. 
milk production decreased significantly as the number of positive tests increased.

\section{DISCUSSION}

This analysis revisited, using updated biological understanding of the MAP infection process, a data set that had previously been analyzed in a similar way (Smith et al., 2009). The predicted difference in milk production shows the importance of considering the eventual outcome of the infection when predicting the effect of JD on milk production.

If path was not considered, as in the previously published analysis of these data, the constant effect of latent and low-status animals was dominated by the (far more common) low-path animals. This masked the fact that the higher production previously observed in latent animals (Nielsen et al., 2009; Smith et al., 2009) is much more pronounced in animals that progress to clinical disease. This production advantage, however, decreases slightly as animals approach their first positive test result. After the first positive test result, both low- and high-path animals showed a mean milk production that was slightly lower than that of testnegative animals, but that difference reduced over time (returning to previous production levels) in low-path animals. This would indicate that low-shedding animals are able to recover milk production levels after a shedding event, a phenomenon not captured by the model that does not include MAPpath (Smith et al., 2009). The path $\times M$ thP interaction was not significant, but subset analysis (data not shown) indicated that recovery was present only in low-path animals; high-path animals continued to show decreasing milk production over time. That is to be expected, as high-shedding animals show dramatically decreased milk production and milk production continues to decrease over time. It has been suggested that the immune systems of highpath animals are unable to mount a sufficient response to control the infection (Stabel, 2000; Mitchell et al., 2015a); our results suggest that those animals also struggle to maintain milk production, as opposed to the low-path animals that are able to recover pre-infection milk production.

It is possible that false-positive results could have caused the recovery in milk production noticed in the low-positive animals, especially due to the repeated testing of animals and the imperfect specificity of the ELISA. However, all ELISA-positive animals in this study were also positive by tissue culture at slaughter, as were all animals low-positive on FC. This indicates that the number of false-positive tests is practically negligible in this population.
The implications of these results for cost-effective control of MAP are large. Low-shedding animals may recover milk production after a shedding event or positive ELISA. These animals, therefore, might continue to contribute to a herd's economic well-being. If an animal continues to show decreasing milk production, however, that may be considered an indication that it is progressing toward clinical disease and increased shedding. Previous studies have shown that factors such as the strength of the ELISA response were able to predict decreases in milk production (Lombard et al., 2005), recommending that this be factored into culling decisions in control programs (Dorshorst et al., 2006). We were unable to include the strength of the ELISA response in our model, as ELISA results were recorded only as positive/negative, but these findings align with ours in that the strength of the fecal culture result was highly associated with the size of the effect on milk production.

One potential indicator of an animal's infection path is its number of positive test results. Our data show that the number of positive tests, particularly the number of positive FC tests, is (1) significantly correlated with the MAP infection path, and (2) is a better predictor of milk production. Although the animals in this study were tested more frequently than would be practical in a commercial dairy herd, the clinical implications of this are important: multiple positive tests are a good indication that an animal will have both a progressing infection and decreased milk production. Therefore, culling could focus simply on the number of positive tests an animal has had rather than whether or not the tests were high- or low-positive. As tests improve, becoming more sensitive and specific, the value of this approach will increase.

This study was conducted in well-managed, lowprevalence herds, with culling programs based on the reported test results. Although this would decrease the number of observations available for analysis, especially among high-shedding animals, it is unlikely that any systematic bias was introduced by this process.

\section{CONCLUSIONS}

This research leads to 3 conclusions. First, culling for a low-positive fecal culture or a single positive ELISA or PCR is not likely to be optimal for maintaining production levels in endemically infected herds. Second, animals with multiple positive test results are likely to have decreased milk production over time and are more likely to have progressing infections than animals with only one positive test result. Third, monitoring the long-term milk production of MAP-positive ani- 
mals may enable herd managers to identify high-path animals before their shedding has progressed; culling of these animals could very well be cost-effective, although the decrease in milk production is not necessarily specific to progressing MAP infection. Economic models of management strategies in MAP-infected herds should account for animals with positive tests either having self-limiting infection or progressing to clinical disease.

\section{ACKNOWLEDGMENTS}

The authors gratefully acknowledge funding provided by the Office of Research Infrastructure Programs (ORIP) of the National Institutes of Health (Bethesda, MD) through Grant Number 8K01OD01968-04 and the National Institute of Food and Agriculture of the United States Department of Agriculture (Washington, DC) through NIFA Award No. 2014-67015-2240.

\section{REFERENCES}

Cho, J., L. W. Tauer, Y. H. Schukken, M. I. Gómez, R. L. Smith, Z. Lu, and Y. T. Gröhn. 2012. Economic analysis of Mycobacterium avium subspecies paratuberculosis vaccines in dairy herds. J. Dairy Sci. 95:1855-1872. http://dx.doi.org/10.3168/jds.2011-4787.

Cho, J., L. W. Tauer, Y. H. Schukken, R. L. Smith, Z. Lu, and Y. T. Gröhn. 2013. Cost effective control strategies for Johne's disease in dairy herds. Can. J. Agric. Econ. 61:583-608. http://dx.doi. org/10.1111/j.1744-7976.2012.01270.x.

Dorshorst, N. C., M. T. Collins, and J. E. Lombard. 2006. Decision analysis model for paratuberculosis control in commercial dairy herds. Prev. Vet. Med. 75:92-122.

Dye, C., and B. G. Williams. 2008. Eliminating human tuberculosis in the twenty-first century. J. R. Soc. Interface 5:653-662. http:/ dx.doi.org/10.1098/rsif.2007.1138.

Gomes, M. G. M., R. Águas, J. S. Lopes, M. C. Nunes, C. Rebelo, P. Rodrigues, and C. J. Struchiner. 2012. How host heterogeneity governs tuberculosis reinfection? Proc. Biol. Sci. 279:2473-2478. http://dx.doi.org/10.1098/rspb.2011.2712.

Gonda, M. G., Y. M. Chang, G. E. Shook, M. T. Collins, and B. W. Kirkpatrick. 2007. Effect of Mycobacterium paratuberculosis infection on production, reproduction, and health traits in US Holsteins. Prev. Vet. Med. 80:103-119. http://dx.doi.org/10.1016/j. prevetmed.2007.01.011

Kudahl, A. B., S. S. Nielsen, and J. T. Sørensen. 2004. Relationship between antibodies against Mycobacterium avium ssp. paratuberculosis in milk and shape of lactation curves. Prev. Vet. Med. 62:119-134. http://dx.doi.org/10.1016/j.prevetmed.2003.11.008.

Liao, C.-M., Y.-J. Lin, and Y.-H. Cheng. 2013. Modeling the impact of control measures on tuberculosis infection in senior care facilities. Build. Environ. 59:66-75. http://dx.doi.org/10.1016/j. buildenv.2012.08.008.

Lombard, J. E., F. Garry, B. J. McCluskey, and B. A. Wagner. 2005. Risk of removal and effects on milk production associated with paratuberculosis status in dairy cows. J. Am. Vet. Med. Assoc. 227:1975-1981.

Meima, A., M. D. Gupte, G. J. van Oortmarssen, and J. D. F. Habbema. 1999. SIMLEP: A simulation model for leprosy transmission and control. Int. J. Lepr. Other Mycobact. Dis. 67:215-236.

Mitchell, R. M., Y. H. Schukken, A. P. Koets, M. F. Weber, D. Bakker, J. R. Stabel, R. H. Whitlock, and Y. Louzoun. 2015a. Differences in intermittent and continuous fecal shedding patterns between natural and experimental Mycobacterium avium subspecies paratuberculosis infections in cattle. Vet. Res. 46:66.

Mitchell, R. M., R. H. Whitlock, Y. T. Grohn, Y. H. Schukken, Y. T. Gröhn, and Y. H. Schukken. 2015b. Back to the real world: Connecting models with data. Prev. Vet. Med. 118:215-225. http:// dx.doi.org/10.1016/j.prevetmed.2014.12.009.

Nielsen, S. S., M. A. Krogh, and C. Enevoldsen. 2009. Time to the occurrence of a decline in milk production in cows with various paratuberculosis antibody profiles. J. Dairy Sci. 92:149-155. http:// dx.doi.org/10.3168/jds.2008-1488.

Ott, S. L., S. J. Wells, and B. A. Wagner. 1999. Herd-level economic losses associated with Johne's disease on US dairy operations. Prev. Vet. Med. 40:179-192.

Pinheiro, J., D. Bates, S. DebRoy, D. Sarkar, and The R Development Core Team. 2015. nlme: Linear and Nonlinear Mixed Effects Models. Accessed Nov. 9, 2015. 2015. https://cran.r-project.org/web/ packages/nlme/nlme.pdf.

Pradhan, A. K., J. S. Van Kessel, J. S. Karns, D. R. Wolfgang, E. Hovingh, K. A. Nelen, J. Smith, R. H. Whitlock, T. L. Fyock, S. Ladely, P. J. Fedorka-Cray, and Y. H. Schukken. 2009. Dynamics of endemic infectious diseases of animal and human importance on three dairy herds in the northeastern United States. J. Dairy Sci. 92:1811-1825. http://dx.doi.org/10.3168/jds.2008-1486.

Smith, R. L., Y. T. Gröhn, A. K. Pradhan, R. H. Whitlock, J. S. Van Kessel, J. Smith, D. R. Wolfgang, and Y. H. Schukken. 2009. A longitudinal study on the impact of Johne's disease status on milk production in individual cows. J. Dairy Sci. 92:2653-2661. http:// dx.doi.org/10.3168/jds.2008-1832.

Smith, R. L., R. L. Strawderman, Y. H. Schukken, S. J. Wells, A K. Pradhan, L. A. Espejo, R. H. Whitlock, J. S. Van Kessel, J. M. Smith, D. R. Wolfgang, and Y. T. Gröhn. 2010. The effect of Johne's disease status on reproduction and culling in dairy cattle. J. Dairy Sci. 93:3513-3524.

Stabel, J. R. 2000. Transitions in immune responses to Mycobacterium paratuberculosis. Vet. Microbiol. 77:465-473. http://dx.doi. org/10.1016/S0378-1135(00)00331-X.

The R Development Core Team. 2014. R: A Language and Environment for Statistical Computing. R Foundation for Statistical Computing, Vienna, Austria.

Whitlock, R. H., and C. D. Buergelt. 1996. Preclinical and clinical manifestations of paratuberculosis (including pathology). Vet. Clin. North Am. Food Anim. Pract. 12:345-356.

Wilmink, J. B. M. 1987. Adjustment of test-day milk, fat, and protein yield for age, season and stage of lactation. Livest. Prod. Sci. $16: 335-348$ 